\title{
QCD Physics from CMS
}

\section{Krisztián Krajczár*ifor the CMS Collaboration}

Eötvös Loránd University, Budapest, Hungary

E-mail: krisztian.krajczarecern.ch

CMS is pursuing a rich program of QCD physics. Initial data collected in 2009 at both $900 \mathrm{GeV}$ and $2.36 \mathrm{TeV}$ has been used for commissioning the detector and for the first measurements of charged hadron pseudorapidity and transverse momentum distributions as well as Bose-Einstein correlations. These first results from CMS at the new energy frontier accessible at the LHC are presented. For non-single-diffractive interactions, the average charged-hadron transverse momentum is measured to be $0.46 \pm 0.01$ (stat.) \pm 0.01 (syst.) $\mathrm{GeV} / c$ at $0.9 \mathrm{TeV}$ and $0.50 \pm 0.01$ (stat.) \pm 0.01 (syst.) $\mathrm{GeV} / c$ at $2.36 \mathrm{TeV}$, for pseudorapidities between -2.4 and +2.4 . At these energies, the measured pseudorapidity densities in the central region, $d N_{\mathrm{ch}} /\left.d \eta\right|_{|\eta|<0.5}$, are $3.48 \pm$ 0.02 (stat.) \pm 0.13 (syst.) and $4.47 \pm 0.04$ (stat.) \pm 0.16 (syst.), respectively. The results at $0.9 \mathrm{TeV}$ are in agreement with previous measurements and confirm the expectation of near equal hadron production in $p \bar{p}$ and $p p$ collisions. The measured strength and effective size (using exponential parametrization) of the Bose-Einstein correlation are $\lambda=0.625 \pm 0.021$ (stat.) \pm 0.046 (syst.) and $r=1.59 \pm 0.05$ (stat.) \pm 0.19 (syst.) fm at $0.9 \mathrm{TeV} ; \lambda=0.663 \pm 0.073$ (stat.) \pm 0.048 (syst.) and $r=1.99 \pm 0.18$ (stat.) \pm 0.24 (syst.) fm at $2.36 \mathrm{TeV}$.

XVIII International Workshop on Deep-Inelastic Scattering and Related Subjects, DIS 2010 April 19-23, 2010

Firenze, Italy

\footnotetext{
* Speaker.

${ }^{\dagger}$ Also at KFKI RMKI, Budapest, Hungary
} 


\section{The CMS Experiment}

A detailed description of the Compact Muon Solenoid (CMS) experiment can be found elsewhere [1]. The central feature of the CMS apparatus is a superconducting solenoid, of $6 \mathrm{~m}$ internal diameter. The silicon pixel and strip tracker, the crystal electromagnetic calorimeter (ECAL) and the brass-scintillator hadronic calorimeter (HCAL) are located within the field. Muons are measured in gas chambers embedded in the iron return yoke. Besides the barrel and endcap detectors, CMS has extensive forward calorimetry. Mid-rapidity charged particles are tracked by three layers of silicon pixel detectors (Pixel), made of 66 million $100 \times 150 \mu \mathrm{m}^{2}$ pixels, followed by ten microstrip layers (SST), with strips of pitch between 80 and $180 \mu \mathrm{m}$.

\section{Charged Hadron Spectra}

The $d N_{\mathrm{ch}} / d \eta$ distributions were obtained using three methods based on counting of (i) reconstructed clusters in the pixel barrel detector; (ii) pixel tracklets composed of pairs of clusters in different pixel barrel layers; and (iii) tracks reconstructed in the full tracker volume, combining the pixel and strip hits. The latter method is capable of reconstructing the $d N_{\mathrm{ch}} / d p_{\mathrm{T}}$ distributions, too.

Pixel cluster counting method The pseudorapidity distribution of primary charged hadrons produced in a pp collision can be measured by counting the number of clusters they create when traversing each of the three pixel barrel layers. The cluster counting method correlates the observed pixel-cluster length in the $z$ direction with the expected path length traveled by a primary particle at a given $\eta$ value. Small clusters at large $|\eta|$ are due to loopers, secondary particles and decay products. These clusters were efficiently removed by a cluster-length cut [2].

Pixel-tracklet method Pixel tracklets are constructed from combinations of two pixel hits in any two pixel barrel layers. The difference in the angular positions of the two clusters with respect to the primary vertex, $\Delta \eta$ and $\Delta \phi$, are calculated for each tracklet. If two tracklets share a hit, the tracklet with the larger $\Delta \eta$ is discarded. Tracklets from primary particles display a sharp peak at $\Delta \eta=0$ and two peaks around $\Delta \phi=0$, while the tracklets from the combinatorial background have an extended tail. The combinatorial background was rejected using a sideband method in $\Delta \phi$ [2].

Vertexing and tracking method In order to reconstruct the primary vertex, tracks consisting of triplets of pixel hits were formed. The vertex-reconstruction algorithm uses the $z$ coordinate of the tracks and the corresponding estimated measurement uncertainty. It performs an agglomerative clustering by adding tracks to form group, which are then merged [3].

Pixel and SST detectors were used to reconstruct tracks, including both barrel and end-cap layers. The applied iterative reconstruction procedure follows [4], but was further optimized for primary-track reconstruction in minimum bias events [2].

Results The measured invariant yields of charged hadrons are shown in the left panel of Fig. 1. The average transverse momentum, calculated from the measured data points adding the low- and high- $p_{\mathrm{T}}$ extrapolations from the fit, is $\left\langle p_{\mathrm{T}}\right\rangle=0.46 \pm 0.01$ (stat.) \pm 0.01 (syst.) $\mathrm{GeV} / c$ for the 0.9 $\mathrm{TeV}$ and $0.50 \pm 0.01$ (stat.) \pm 0.01 (syst.) $\mathrm{GeV} / c$ for the $2.36 \mathrm{TeV}$ data. 

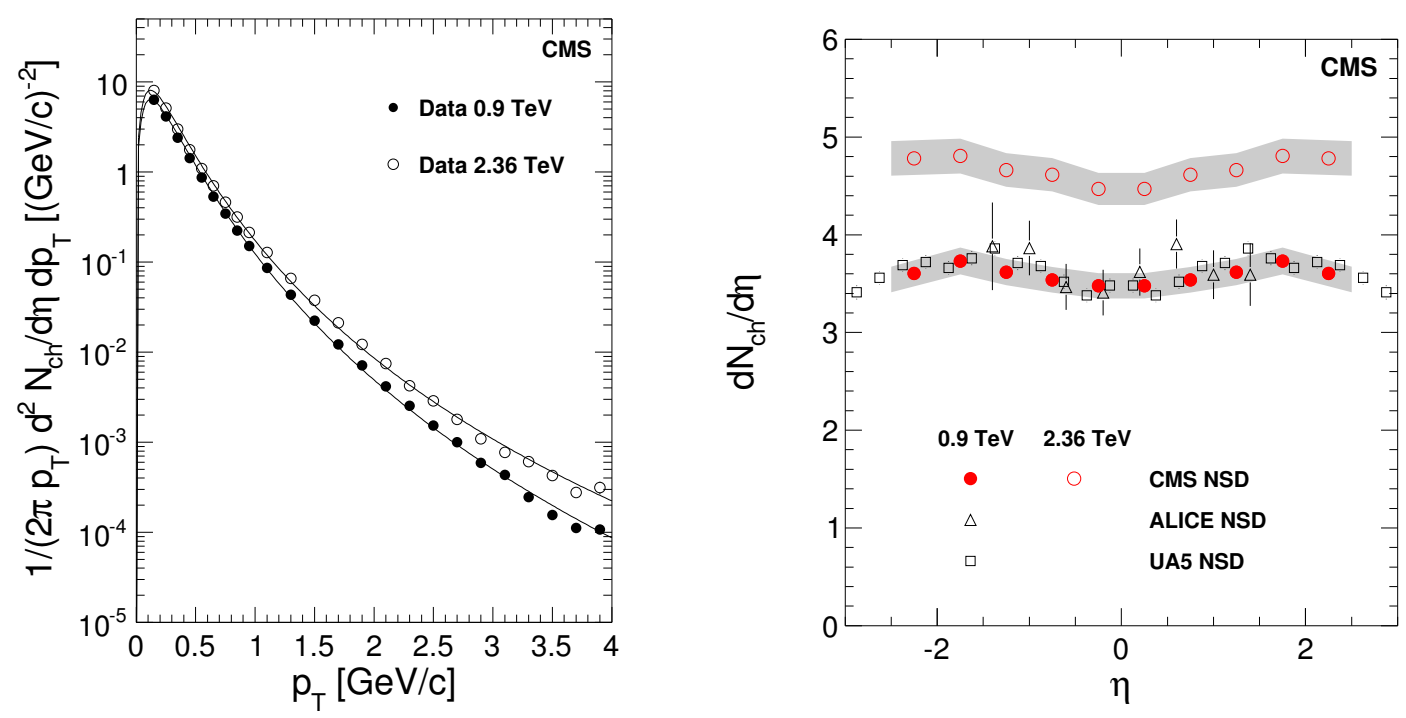

Figure 1: Left: Measured yield of charged hadrons for $|\eta|<2.4$ with systematic uncertainties (symbols), fit with the Tsallis function [5]. Right: Reconstructed $d N_{c h} / d \eta$ distributions averaged over the cluster counting, tracklet and tracking methods (circles), compared to data from previous experiments at $0.9 \mathrm{TeV}$, and the averaged result over the three methods at 2.36 TeV (open circles) (detailed references are in [2]). The shaded band represents systematic uncertainties of this measurement. The error bars on the UA5 and ALICE data points are statistical only.

The $d N_{\mathrm{ch}} / d \eta$ distribution was calculated as the weighted average of the data from the three reconstruction methods. The averaged result is shown in the right panel of Fig.1. For $|\eta|<0.5$, the corrected results average to $d N_{\mathrm{ch}} / d \eta=3.48 \pm 0.02$ (stat.) \pm 0.13 (syst.) and $d N_{\mathrm{ch}} / d \eta=$ $4.47 \pm 0.04$ (stat.) \pm 0.16 (syst.) for NSD events at $\sqrt{s}=0.9$ and $2.36 \mathrm{TeV}$. The increase of $(28.4 \pm 1.4 \pm 2.6) \%$ from 0.9 to $2.36 \mathrm{TeV}$ is significantly larger than the $18.5 \%$ (14.5\%) increase predicted by the PYTHIA 6.420 D6T tune (PHOJET 1.12-35) used in this analysis [6, 7].

The collision energy dependence of the measured $\left\langle p_{\mathrm{T}}\right\rangle$ and $d N_{\mathrm{ch}} /\left.d \eta\right|_{\eta \approx 0}$ is shown in Fig. 2.

\section{Bose-Einstein Correlations}

In particle collisions, the space-time structure of the hadronization source can be studied using measurements of Bose-Einstein correlations (BEC) between pairs of identical bosons

Constructive interference affects the joint probability for the emission of a pair of identical bosons with four-momenta $p_{1}$ and $p_{2}$. Experimentally, the proximity in phase space between finalstate particles is quantified by the Lorentz-invariant quantity $Q=\sqrt{-\left(p_{1}-p_{2}\right)^{2}}=\sqrt{M^{2}-4 m_{\pi}^{2}}$, where $M$ is the invariant mass of the two particles, assumed to be pions with mass $m_{\pi}$. The BEC effect is observed as an enhancement at low $Q$ of the ratio of the $Q$ distributions for pairs of identical particles in the same event, and for pairs of particles in a reference sample that by construction is expected to include no BEC effect: $R(Q)=(d N / d Q) /\left(d N_{\text {ref }} / d Q\right)$, which is then fitted with the parameterization $R(Q)=C[1+\lambda \Omega(Q r)](1+\delta Q)$. 

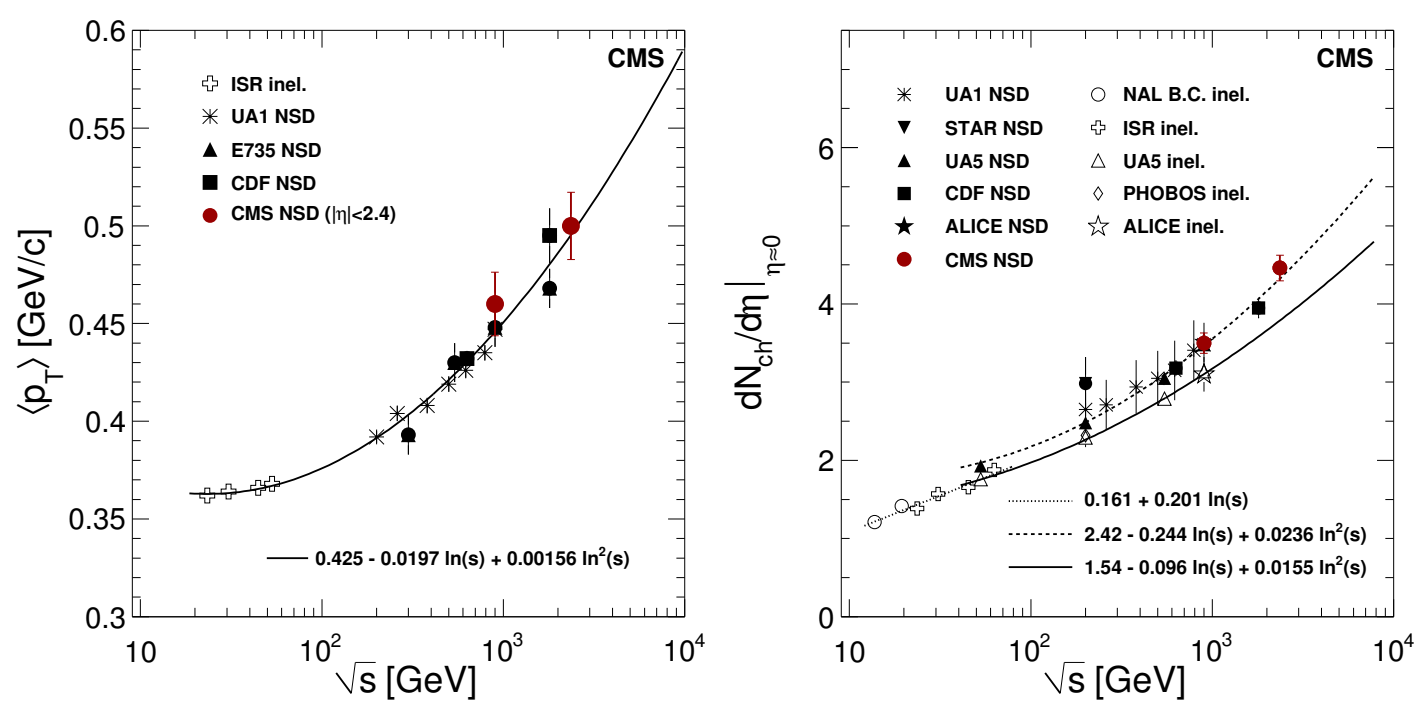

Figure 2: $p p$ and $p \bar{p}$ collision results as a function of centre-of-mass energy, detailed references can be found in [2]. Left: Energy dependence of the average transverse momentum of charged hadrons. The CMS data points are evaluated for the range $|\eta|<2$.4. The error bars on the CMS data points include systematic uncertainties. Right: Charged-hadron pseudorapidity density in the central region as a function of centre-ofmass energy . The error bars indicate systematic uncertainties, when available.

$\Omega(Q r)$ is the Fourier transform of the spatial distribution of the emission region of bosons with overlapping wave functions, characterized by an effective size $r$. The parameter $\lambda$ reflects the BEC strength for incoherent boson emission from independent sources, $\delta$ accounts for long-range momentum correlations, and $C$ is a normalization factor.

Experimental methods Different methods are designed to pair uncorrelated charged particles and to define reference samples used to extract the reference distribution in the expression of $R(Q)$. Opposite-charge pairs: this data set is a natural choice but contains resonances $(\eta, \rho, \ldots)$ which are not present in the same-charge combinations. Opposite-hemisphere pairs: tracks are paired after inverting the three-momentum of one of the two particles. Rotated particles: particle pairs are constructed after inverting the $x$ and $y$ components of the three-momentum of one of the two particles. Pairs from mixed events: particles from different events are combined with the following methods: i) events are mixed at random; ii) events with similar charged particle multiplicity in the same $\eta$ regions are selected; iii) events with an invariant mass of all charged particles similar to that of the signal are used to form the pairs.

As none of the definitions of the reference samples is preferable a priori, an additional, "combined" double ratio $\mathscr{R}^{\mathrm{comb}}$ is formed, where the data and MC distributions are obtained by summing the $Q$ distributions of the seven corresponding reference samples [8].

Results The distributions of $\mathscr{R}^{\text {comb }}$ for 0.9 and $2.36 \mathrm{TeV}$ data are shown in the left panel of Fig.3. The data are well described with an exponential form for $\Omega(Q r)$ (solid lines), while a Gaussian form (dashed lines) does not correctly describe it. 

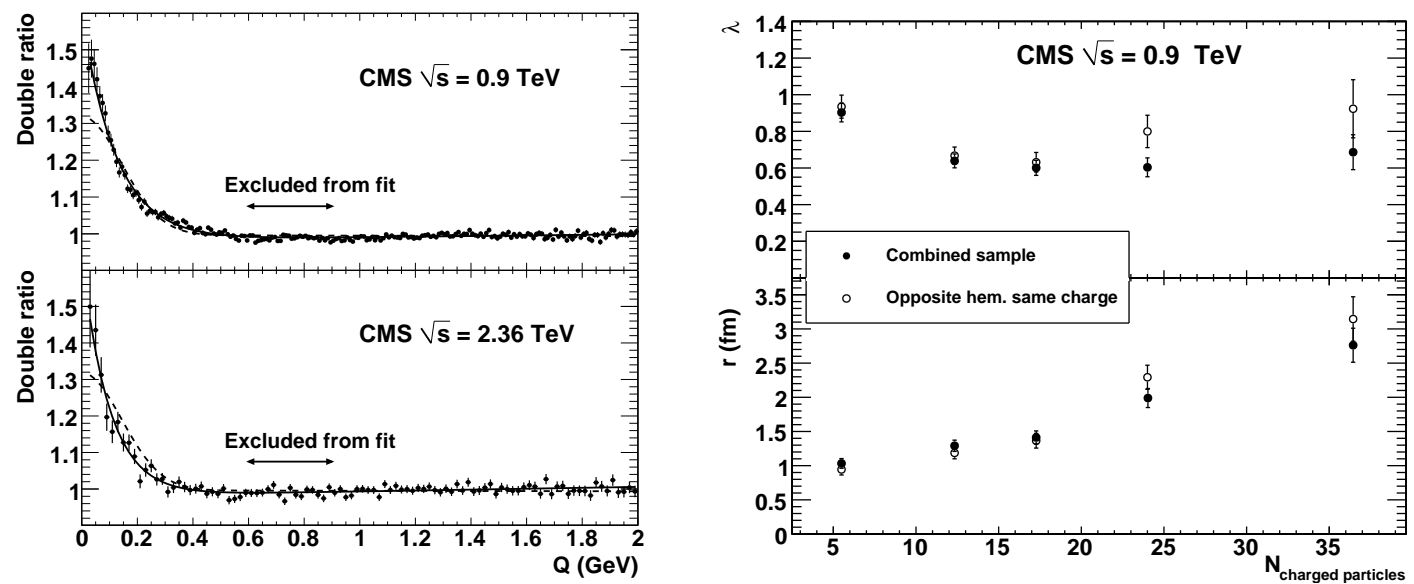

Figure 3: Left: Fits to the double ratios $\mathscr{R}^{\mathrm{comb}}(Q)$ with exponential (solid lines) and Gaussian (dashed lines) functions, for $0.9 \mathrm{TeV}$ (top) and $2.36 \mathrm{TeV}$ (bottom) data. The range corresponds to the $\rho$ resonance, $0.6<Q<0.9 \mathrm{GeV}$, is excluded from the fits. Right: Values of the $\lambda$ (top) and $r$ (bottom) parameters as a function of the charged-particle multiplicity in the event for combined (dots) and opposite-hemisphere, same-charge (open circles) reference samples, at $0.9 \mathrm{TeV}$. The errors shown are statistical only.

The BEC parameters measured with the combined reference sample are $\lambda=0.625 \pm 0.021$ (stat.) \pm 0.046 (syst.) and $r=1.59 \pm 0.05$ (stat.) \pm 0.19 (syst.) fm at $0.9 \mathrm{TeV} ; \lambda=0.663 \pm 0.073$ (stat.) \pm 0.048 (syst.) and $r=1.99 \pm 0.18$ (stat.) \pm 0.24 (syst.) fm at $2.36 \mathrm{TeV}$ [8].

The right panel of Fig. 3 shows these parameters as a function of track multiplicity at $0.9 \mathrm{TeV}$.

\section{Acknowledgments}

The author wishes to thank the Hungarian Scientific Research Fund, the National Office for Research and Technology and the Swiss National Science Foundation (K 81614, NK 81447, NKTH-OTKA H07-C 74248 and 128079) for their support.

\section{References}

[1] CMS Collaboration, The CMS experiment at the CERN LHC, JINST 0803 (2008) S08004.

[2] CMS Collaboration, Transverse-momentum and pseudorapidity distributions of charged hadrons in pp collisions at $\sqrt{s}=0.9$ and $2.36 \mathrm{TeV}$, JHEP 02 (2010) 041

[3] F. Sikler, Improved primary vertex finding for collider detectors, accepted by NIM A, arXiv:0911.2767

[4] T. Speer et al., Track reconstruction in the CMS tracker, Nucl. Instrum. and Methods A559 (2006) 143

[5] T. Biro at al., A non-conventional description of quark matter, J. Phys. G31 (2005) S759-S763

[6] T. Sjöstrand et al., PYTHIA 6.4 Physics and Manual; v6.420, tune D6T, JHEP 05 (2006) 026

[7] F. W. Bopp et al., Rapidity gaps and the PHOJET Monte Carlo, arXiv:hep-ph/9803437

[8] CMS Collaboration, First Measurement of Bose-Einstein Correlations in proton-proton Collisions at $\sqrt{s}=0.9$ and $2.36 \mathrm{TeV}$ at the LHC, arXiv:1005.3294 